\title{
Lidil
}

Revue de linguistique et de didactique des langues

$31 \mid 2005$

Corpus oraux et diversité des approches

\section{Les interactions pédagogiques comme polylogues}

\section{Robert Bouchard}

\section{OpenEdition}

\section{Journals}

Édition électronique

URL : http://journals.openedition.org/lidil/150

DOI : $10.4000 /$ lidil. 150

ISSN : 1960-6052

\section{Éditeur}

UGA Éditions/Université Grenoble Alpes

\section{Édition imprimée}

Date de publication : 1 juin 2005

Pagination : 139-155

ISBN : 2-914176-12-0

ISSN : $1146-6480$

Référence électronique

Robert Bouchard, «Les interactions pédagogiques comme polylogues », Lidil [En ligne], 31 | 2005, mis en ligne le 03 octobre 2007, consulté le 01 mai 2019. URL : http://journals.openedition.org/lidil/150 ; DOI : $10.4000 /$ lidil. 150

Ce document a été généré automatiquement le 1 mai 2019.

(C) Lidil 


\title{
Les interactions pédagogiques comme polylogues
}

\author{
Robert Bouchard
}

1 L'étude pragmatique des interactions pédagogiques "ordinaires" implique de s'intéresser à des « leçons » en tant qu' « incursions » (Roulet et al., 1985) pédagogiques de base autant sinon plus qu'à des moments pédagogiques particuliers (correction d'exercice, interrogation d'élève au tableau...). Cela demande de prendre en compte l'ensemble des participants de ces leçons, l'enseignant et les élèves dans leur individualité. Nous développerons en conséquence la problématique du polylogue, problématique encore assez peu prise en compte (Kerbrat-Orecchioni, 2004) dans les travaux interactionnels. Ajoutons que, dans la mesure où ces évènements interactionnels mêlent actions verbales et actions non verbales, nous nous situons dans une pragmatique de la "quatrième génération' », négociant son virage actionnel (Filliettaz, 2002), même si nous n'aurons pas le temps de traiter cet aspect de la question ici-mêmé2.

2 Nous essayons d'adopter dans ce travail une attitude de pragma-didacticien (cf. aussi Sensevy \& Quillio 2002 pour un projet de « pragmatique didactique »). Cela veut dire que nous travaillons dans le champ didactique en général - et en particulier dans celui de la didactique des langues - avec des outils pragmatiques adaptés (Bouchard, 1998, 1999, $2004 \mathrm{~b}$ et c; Bouchard \& Rolet, 2003). Cet intérêt, que l'on pourrait qualifier d'« interdidactique» est motivé par deux raisons. D'une part il s'agit pour nous de nous rapprocher du point de vue de l'élève d'âge scolaire qui doit s'adapter perpétuellement aux changements de discipline et aux variations éventuelles des normes interactionnelles correspondantes. D'autre part en tant que didacticien de la langue maternelle, qui est aussi celle de l'école, il nous semble intéressant d'observer son rôle non seulement comme objet spécifique d'apprentissage mais aussi comme moyen général d'apprentissage : les interactions de classe ne peuvent qu'être au centre de l'intérêt de toutes les didactiques des disciplines. Par ailleurs nous restons persuadés que ces interactions pédagogiques dans leur spécificité permettent un élargissement de l'expérience de la "pragmatique interactionnelle » longtemps confinée à des situations trop purement conversationnelles ou en tout cas dialogales. 
3 Après avoir tracé à grands traits une histoire rapide et un « portrait » du polylogue en général, nous décrirons les manifestations polylogales les plus importantes des échanges pédagogiques pour nous consacrer ensuite aux manifestations polylogales et polylogiques propres aux interventions et/ou aux tours de parole de l'enseignant.

Dialogue : dilogue, trilogue... polylogue

On pourrait décrire l'itinéraire qui mène, en linguistique française, le chercheur à l'étude du polylogue comme la poursuite logique de celui qui a fait s'intéresser progressivement les spécialistes contemporains de l'oral, à la langue (Blanche-Benveniste, 1997), puis au fonctionnement contextuel des discours monologaux (Kerbrat-Orecchioni, 1990a), puis à leur réalisation interactionnelle la plus fréquente, la conversation (Roulet et al., 1985; Kerbrat-Orecchioni, 1990b), et enfin à d'autres réalisations moins fréquentes peut-être mais d'une incontestable importance professionnelle ou sociétale, les polylogues...

Une étape a été marquée avec des travaux sur les trilogues (Kerbrat-Orecchioni \& Plantin, 1995). Ils ont un intérêt stratégique dans la mesure où ils font pour la première fois accomplir aux chercheurs un pas hors du dilogue et de sa stabilité structurale. En effet à partir du trilogue disparait le principe de l'alternance réglée des tours de parole entre les participants, base de l'analyse de la conversation de type ethnométhodologique par exemple. Mais nous y revenons dans la mesure aussi où, comme le trilogue peut être réduit à un dilogue mettant face à face un participant et la " coalition » constituée par les deux autres (Caplow, 1971; Witko-Commeau, 1995), le polylogue peut être ramené à un trilogue constitué - dans le domaine des interactions didactiques par exemple (Schubauer-Léoni, 1997) - d'un dilogue enseignant - apprenant (interrogé), augmenté d'un troisième participant collectif, le reste de la classe. Le trilogue deviendrait alors le modèle, simplifié et économique pour le chercheur de toutes les interactions qui excèdent le dilogue.

6 Cette réduction, pratique pour le chercheur, me semble cependant discutable si on rappelle le cadre participatif de la classe. on oppose souvent de ce point de vue, les participants ratifiés et non-ratifiés de l'interaction. Force alors est de constater qu'en classe tous les élèves sont a priori des participants ratifiés même si un interlocuteur peut être plus « visé » à un certain moment que les autres, avec tous les tropes communicatifs envisageables (à bon entendeur salut!). Un participant non ratifié serait un auditeur accidentel ou intrusif, assez difficile à concevoir dans l'espace institutionnellement clos de la classe (on peut envisager cependant les cas marginaux de l'inspecteur ou du... chercheur!).

7 Enfin rappelons d'un point de vue plus didactique, que le but de l'interaction pédagogique est que... tous les apprenants présents apprennent. Ce but est visé par tous les maîtres. On peut postuler également qu'il est partagé par tous les élèves, ayant conclu a priori le même " contrat didactique ». Que ces élèves soient interrogés ou non-interrogés, ils sont toujours présents et en situation d'action conjointe sinon de co-action (Vernant, 1997). La prise en compte effective du nombre de participants nous semble donc objectivement la solution la meilleure car à la fois la plus respectueuse des données et à terme la plus fiable dans le traitement pragma-didactique de ces données.

8 Ajoutons pour finir que cette première opposition dialogale de types "matériellement ", quantitativement, différents, se redouble d'une opposition dialogique, de nature énonciative prenant en compte le nombre et la nature des voix que font entendre les tours de parole (Roulet et al., 1985). Nous verrons que ce jeu des voix est particulièrement 
important dans le travail d'hétéro-reformulation qui fonde une part importante du dialogue didactique (Rabatel, 2004).

Les manifestations polylogales dans la classe ordinaire

9 Toute interaction mettant en jeu plus de deux participants est relativement instable : la règle de l'alternance des tours de parole entre deux interlocuteurs ne peut plus fonctionner. Cette instabilité apparaît dès le trilogue (Caplow, 1971). Elle est donc constitutive du polylogue et nécessite pour les polylogues institutionnels une organisation réglée, spécifique des échanges qui le constituent et une distribution précise des rôles des participants. L'enseignant par son âge, son statut, sa détention de savoirs devient (entre autres fonctions) le maitre de cérémonie indispensable du polylogue pédagogique. Il peut le réduire à une pratique monologale ostensive comme le cours magistral. Le professeur énonce alors un discours préparé à l'avance en le mettant en voix et en scène dans une performance quasi théâtrale. Cette pratique (Bouchard, Parpette \& Pochard, 2005) réservée à l'enseignement supérieur est en général remplacée dans l'enseignement secondaire et primaire par le cours dialogué étudié tout d'abord par Sinclair et Coulthard (1975).

Échange ternaire et polylogue

Son élément central est l'échange ternaire, repéré également par Mehan (1978) et plus tard systématisé pour tous les échanges conversationnels par Roulet et al. (1985) qui le définit comme la plus petite unité dialogale constituée de trois unités monologales, les interventions, dont seule la seconde laisse la parole aux élèves :

\begin{tabular}{|l|l|l|l|}
\hline 182 & P & alors livre page 119 chut vous l'avez en couleurs ce plan et il est extrait \\
\hline & & heu de quel livre heu ce plan chut on lève bien le doigt... heu david \\
\hline \hline 183 & Da & asterix légionnaire \\
\hline \hline 184 & $P$ & asterix légionnai:re donc il est extrait d'une bande dessinée \\
\hline
\end{tabular}

Dans le cours frontal classique de l'enseignement secondaire en particulier (le maître «face à » la classe), on retrouve cet échange ternaire dans toutes les disciplines et sous divers cieux pédagogiques, avec des infléchissements mineurs, "simples» adaptations aux conditions matérielles et culturelles d'intervention de l'enseignant. Cette constance le désigne comme caractéristique du traitement didactique d'une interaction polylogale. Il permet une alternance systématique entre le polylogue cadre et de courts moments de dilogue ostensif. On peut qualifier ces derniers de subordonnés, dans la mesure où comme dans l'exemple ci-dessous, ils sont elliptiques et empruntent en particulier leur acte directeur aux interventions polylogales initiatives qui les précèdent au sein de la même prise de parole de l'enseignant. Ces interventions polylogales de l'enseignant se situent dans la première et la troisième intervention, celles qui ont le pouvoir d'initier et de clore l'échange. On les trouve même en miroir sur la «face externe » de ces interventions, au 
début de la première et à la fin de la troisième. Si on examine la suite des échanges pédagogiques, on constate donc que souvent un même tour de parole de l'enseignant associe en les reliant de manière plus ou moins explicite, une intervention de clôture polylogale d'un premier échange et l'intervention d'ouverture de l'échange qui le suit :

\begin{tabular}{|l|l|l|l|l|}
\hline 182 & P & alors livre page 119 chut vous l'avez en couleurs ce plan \\
\hline & & et il est extrait heu de quel livre heu ce plan chut on lève bien le doigt... heu \\
\hline \hline & & david \\
\hline 183 & E & asterix légionnaire \\
\hline 184 & P & oui asterix légionnai:re \\
\hline \hline & & donc il est extrait d'une bande dessinée \\
\hline \hline & & & \\
\hline
\end{tabular}

Seul le cœur (caractères romain-maigre) de cet épisode pédagogique minimal est de nature dilogale. On trouve d'ailleurs des corpus pédagogiques francophones (Afrique du Nord) où ce moment dilogal peut disparaître. L'épisode minimal se réduisant alors monologiquement, à une question initiale, de type rhétorique, d'« alerte thématique ", suivie, après une légère pause de l'apport de l'information « officielle » par le maître, sur le schéma :

Exemple $1 \mathrm{~b}$ : (exemple artificiel)

\begin{tabular}{|l|l|}
\hline P & alors livre page 119chut vous l'avez en couleurs ce plan \\
\hline \hline & et il est extrait heu de quel livre heu ce plan chut \\
\hline \hline & (temps de réflexion) \\
\hline \hline 2 P & il est extrait d'une bande dessinée \\
\hline
\end{tabular}

Il s'agit bien alors d'un moyen de différer l'apport d'information.

On peut trouver dans d'autres pays francophones une amplification trilogale de l'évaluation magistrale dilogale (ci-dessus $186 \mathrm{P}$ : oui asterix légionnaire). L'ensemble des 
élèves non-interrogés est prié par le maître d'applaudir la bonne réponse de l'élève interrogé (Noyau \& Quashié, 2004, pour le Mali).

\begin{tabular}{|l|l|l|l|l|}
\hline & $\mathrm{P}$ & $\mathrm{EE}$ & (sollicitation implicite par le maître) \\
\hline & $\mathrm{EE}$ & $\mathrm{P}$ & (demandes de parole simultanées) \\
\hline
\end{tabular}

21 La première intervention de l'échange est donc le lieu privilégié d'organisation locale du polylogue, le moment où se gère à chaque nouvel échange une nouvelle distribution de la parole, la mise en scène d'un nouveau dilogue public ostensif momentané. Cette phase technique peut être elle aussi simplifiée, le maître ayant le droit de choisir lui-même son interlocuteur, y compris, éventuellement, parmi les élèves non volontaires...

Exemple 2 : (Histoire $\left.6^{\mathrm{e}}\right)$ 


\begin{tabular}{|c|c|c|}
\hline $91 \mathrm{P}$ & & EE la décapitation \\
\hline & & alors on écrit cela s'appelle (3s) la dé-ca-pi-ta-tion (4s) \\
\hline & & $\begin{array}{l}\text { alors là évidemment. ben c'est la peine de mort on le voit bien on lui tranche la } \\
\text { tête }\end{array}$ \\
\hline & & $\begin{array}{l}\text { mais on voit qu'il y a d'autres punitions et parmi ces punitions vous vous } \\
\text { souvenez on }\end{array}$ \\
\hline & & en avait vu une qui était aussi très cruelle laquelle \\
\hline & $\mathrm{Ab}$ & ABDEL KARIM/ \\
\hline $\begin{array}{l}92 \\
\mathrm{Ab}\end{array}$ & $\mathrm{P}$ & ben on les fait sortir du campement tout seul \\
\hline $93 \mathrm{P}$ & $\mathrm{EE}$ & voilà on les fait sortir du campement la nuit \\
\hline
\end{tabular}

\begin{tabular}{|l|l|l|}
\hline & Aj & JoHn et ils pouvaient ben \\
\hline \hline $94 \mathrm{Aj}$ & P & être [attaqués \\
\hline \hline $95 \mathrm{~A} 2$ & P & [être attaqués \\
\hline \hline $96 \mathrm{P}$ & EE & être attaqués et donc être... tués \\
\hline
\end{tabular}

23 Si la question polylogale précède le plus souvent l'adressage dilogal, ce n'est pas toujours le cas comme ci-dessus pour John (en 93 P) où, d'entrée, la question est adressée à ce seul élève.

24 À travers ces exemples, on constate donc, que, dans ce qu'il est convenu d'appeler un même tour de parole, on peut faire la distinction entre deux interventions (?) qui se succèdent et qui divergent non pas tant par leur valeur illocutoire que par leur fonction perlocutoire. Leur adressage à l'ensemble des élèves puis à un élève particulier donne une simple valeur perlocutoire d'alerte thématique générale à la première et un effet d'intimation individuelle publique à la seconde (avec toutes les conséquences sociales éventuelles de perte de face que peut engendrer alors un comportement de réponse jugé inadéquat).

Ajoutons enfin que cette phase d'attribution de la parole qui peut se manifester uniquement par un geste ou un regard est l'occasion aussi d'un éventuel rappel du rituel de demande de parole (cf. exemple 2 ci-dessus). Ces rappels « disciplinaires » fréquents des droits et devoirs de parole (et donc de silence) montrent par leur fréquence même 
l'accroissement de l'instabilité du polylogue qui se manifeste dans ces moments de changement de format interlocutif.

Le triple feuilletage du tour de parole magistral

Du fait de la situation polylogale, on peut parler d'une complexification simultanée des tours de parole magistraux. On constate un triple feuilletage potentiel des tours de parole de l'enseignant. On a déjà vu ci-dessus qu'ils associaient fréquemment deux interventions appartenant de fait à deux échanges différents de l'interaction pédagogique (cf. $186 \mathrm{P}$ dans l'exemple 2a ci-dessus où les deux interventions étaient explicitement articulées : coordonnées par la conjonction "et»). Nous verrons qu'ils sont aussi scindés par des changements de format d'interlocution en même temps que fissurés par d'importantes variations de prise en charge énonciative.

Le feuilletage interlocutif

$27 \mathrm{Au}$ fait du tour de parole de l'enseignant s'opèrent de fréquents changements d'adressage. L'instabilité verbale et actionnelle du polylogue occasionne des réajustements constants du format d'interlocution des interventions de l'enseignant. L'événement de parole polylogal est sujet à des incidents eux-mêmes polylogaux, c'est à dire trouvant leur origine dans les comportements verbaux ou non verbaux de l'un quelconque $\mathrm{X}$ des participants ratifiés :

Exemple 3 : (Mathématiques 6e)

P ChuUUt (3s) ON NE PEUt PAS tRAVAILler COMME ÇA JONATHAN ton livre est toujours pas ouvert alors j'ai demandé le livre ouvert à la page 216 et vos figures devant vous (4s) jeremy tu t'retournes ... christophe nous dit que le premier c'est-à-dire celui qui est en violet dans notre livre ne marche pas

Pendant les cours, au sein d'une même prise de parole, d'une part le maître s'adresse souvent à l'ensemble du groupe (exemple ci-dessous : éléments en caractères gras) mais d'autre part il intercale, si besoin est, des interventions destinées à des individus particuliers explicitement interpellés. Ajoutons que même si ces interventions sont à visée individuelle, il n'est pas mauvais que les autres élèves les entendent dans la mesure où elles rappellent des règles de la vie commune, valables pour tous. On pourrait parler de métonymie conversationnelle (ce qui est adressé à une partie de l'assistance vaut aussi pour la totalité de cette assistance)!

\begin{tabular}{|l|l|l|l|}
\hline P & EE & CHUUUT (3s) ON NE PEUT PAS TRAVAILLER COMME ÇA \\
\hline \hline Jo & & JonATHAn ton livre est toujours pas ouvert \\
\hline \hline & & EE & alors j'ai demandé le livre ouvert à la page 216 et vos figures devant vous \\
\hline \hline & & (4s) \\
\hline \hline & Je & jeremy tu t'retournes... \\
\hline & EE & $\begin{array}{l}\text { christophe nous dit que le premier c'est-à-dire celui qui est en violet dans notre livre } \\
\text { ne }\end{array}$ \\
\hline
\end{tabular}


L'unité monologale est donc en fait constituée d'un ensemble aléatoire d'échanges dilogaux ponctuels et largement indépendants, de nature mixte verbale/non-verbale :

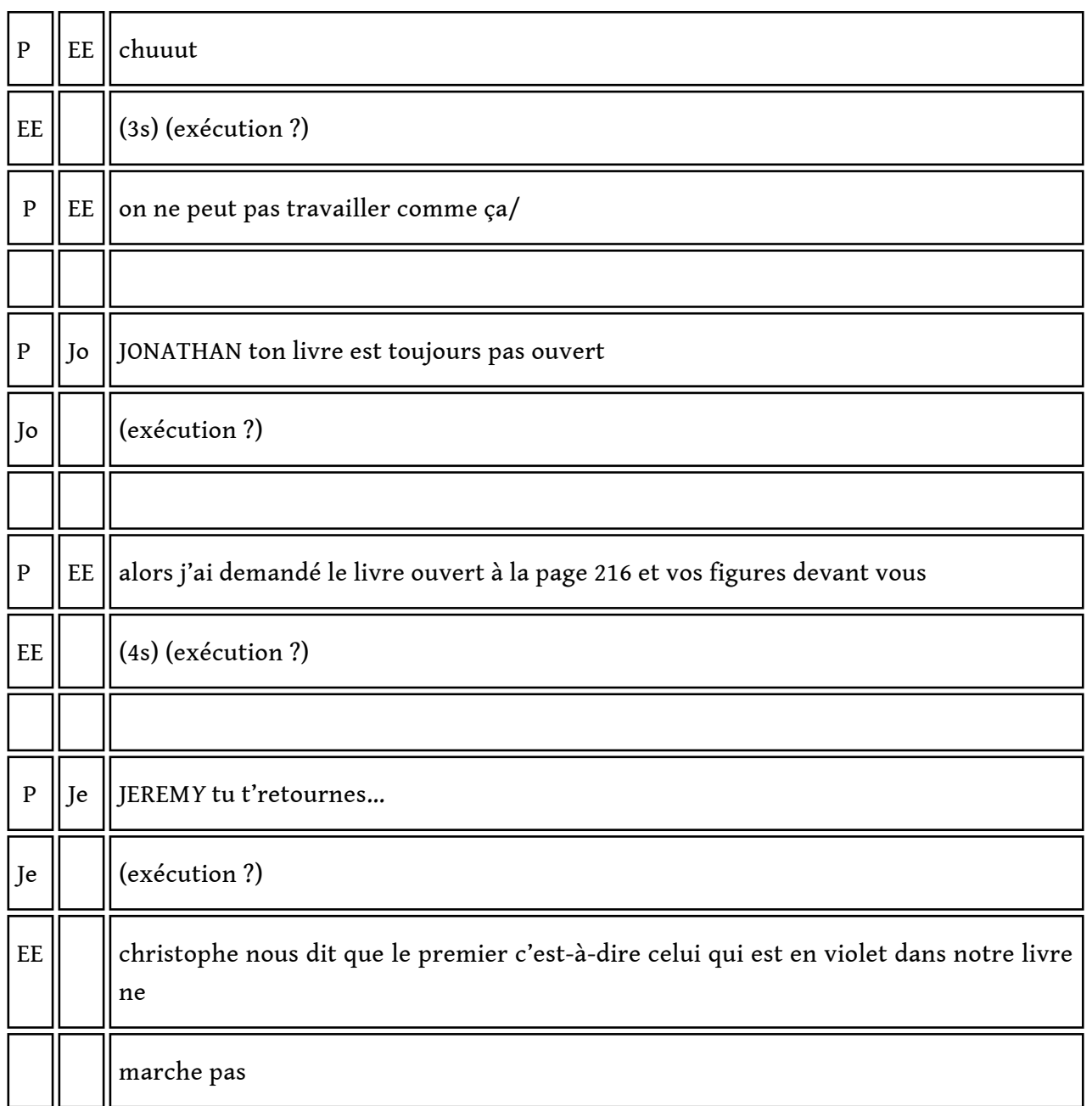

Il s'agit de faire taire un groupe d'élèves, de faire ouvrir son livre par un enfant particulier, faire se retourner un autre pour introduire une nouvelle activité engageant le groupe classe tout entier. De notre point de vue on assiste donc, en situation polylogale, à un ébranlement des notions classiques de tour de parole ou même d'intervention, postulées à partir de corpus dilogaux. Ces notions postulent traditionnellement dans le dilogue une homogénéité illocutoire interne alors qu'ils présentent une hétérogénéité interlocutoire et praxéologique importante.

L'exemple ci-dessus montre autre chose : ce changement d'adressage est dû pour une bonne part à la polyfonctionnalité de ces tours de parole. Ces poly-adressages plus occasionnels sont dus à la mise en œuvre explicite de la dernière des trois grandes fonctions, information, évaluation, organisation, que l'enseignant assure simultanément dans la salle de classe (Dabène, 1984). Des trois c'est en effet celle qui varie en importance 
en fonction de l'importance numérique des participants et donc des incidents de participation qu'ils peuvent occasionner.

Le feuilletage polylogique

Les tours de parole manifestent également une fondamentale hétérogénéité énonciative qui est liée pour une part à la variation du format interlocutoire. Celle-ci se manifeste de manière aléatoire dans l'exemple 3 ci-dessus: l'utilisation du discours rapporté (et éventuellement auto-rapporté), la variation « on »/« nous »/《je » vs « vous »/« tu ». sur votre cahier là on va travailler en 3D d'accord/. bon (3s) celle sur q- laquelle vous avez travaillé dans les activités préparatoires elle s'appelle parallélépipède rectangle comme c'est très très compliqué à écrire ils ont eu la bonne idée de lui donner un autre nom

Exemple 3c : (Histoire 6 ${ }^{\mathrm{e}}$ ):

P CHUUUt (3s) ON NE PEUT PAS TRAVAILLER COMME ÇA JONATHAN ton livre est toujours pas ouvert alors j'ai demandé le livre ouvert à la page 216 et vos figures devant vous (4s) jeremy tu t'retournes... christophe nous dit que le premier c'est à dire celui qui est en violet dans notre livre ne marche pas

Elle est plus fondamentale dans l'exemple 4 ci-dessous où se manifeste, avec quatre items et quatre degrés, une large partie de l'éventail possible de variation de la prise en charge énonciative de ses énoncés par l'enseignant. Soit il peut assumer ses dires en tant qu'adulte responsable du groupe classe résumant le passé collectif de ce groupe (passage en italiques). Soit il peut s'engager plus individuellement en employant des métaphores moins partagées par sa collectivité scientifique adulte ou des jugements de valeur (passage en italiques + gras). Soit au contraire il peut prendre de la distance en neutralisant sa propre parole, lors d'une dénomination scientifique (en caractères romain-maigre) ou d'une définition. Soit enfin il peut attribuer son dire à une autre source générique, un "ils", représentant les auteurs du savoir à enseigner qu'il ne fait que re-produire devant sa classe (Bouchard, 2004 a) :

Exemple 4 : (mathématiques $6^{\mathrm{e}}$ ) :

P solides...

A pa-vé

Mais, plus rarement, l'enseignant peut aller pédagogiquement jusqu'au témoignage personnel, ce qui ajouterait un cinquième degré à notre éventail de degrés de prise en charge :

Exemple 5 : (mathématiques $6^{\mathrm{e}}$ )

P oui alors chut..le verbe de commande qui est utilisé jeremy a raison c'est i-ma-gi-nons que l'on découpe . ALORS MOI ÇA FAIT LONGTEMPS QUAND MÊME QUE JE SUIS PROF DE MATH ET J'PEUX VOUS DIRE UNE CHOSE C'EST QUE... pour imaginer quand c'est en 3D on est pas tous égaux .. y'en a qui arrivent très bien mais y'en a qui arrivent pas et ça n'a rien à voir avec le niveau en mathématique ... MOI. JE SUIS PROFESSEUR DE MATHÉMATIQUE ET J'AI BEAUCOUP DE PEINE À IMAGINER DANS L'ESPACE PAR EXEMPLE

\begin{tabular}{|l|l|l|l|l|}
\hline 1 & 2 & 3 & 4 & 5 \\
\hline
\end{tabular}




\begin{tabular}{|l|l|l|l|l|l|}
\hline Témoignage & Prise en charge & Prise en charge & Neutralisation & Indication d'une \\
\hline \hline didactique $>$ & personnalisée $>$ & hic et nunc > & énonciative > & autre source \\
\hline \hline individuel & & & & \\
\hline \hline & & & & & énonciative \\
\hline
\end{tabular}

prise de parole de plusieurs individus mais aussi au règlement a posteriori de la variété des incidents due à la co-présence active de tous ces participants. Cette complexité nous semble caractéristique de la nature manifestement polylogale de la communication pédagogique. Toute description qui ne prend pas en compte cette nature polylogale nous semble prendre le risque d'oublier volontairement de prendre en compte ce qui fait le propre de la chronogenèse et de la topogenèse didactique.

51 Cette complexité polylogale se combine d'autre part à la pluralité des fonctions correspondant au métier d'enseignant. C'est ce qui différencie sans doute le polylogue pédagogique du polylogue d'une assemblée politique par exemple moins hétérogène de ce dernier point de vue. 
celui classique de polyphonie nous semble rendre compte fonctionnellement de la source du phénomène, la nature polylogale de l'intervention pédagogique.

\section{BIBLIOGRAPHIE}

BLANCHE-BENVENISTE, C. (1997) : Approches de la langue parlée en français, Paris, Ophrys.
BOUCHARD, R. (1998) : L'interaction en classe comme interaction praxéologique, in Grossmann, F (ed.) Pratiques langagières et didactiques de l'écrit, Ivel-Lidilem, Université Stendhal-Grenoble 3, 193-210.

BOUCHARD, R. (1999) : L'interaction pédagogique : unités pragmatiques et phénomènes énonciatifs, in Barberis, J.M. (ed.) Le français parlé variétés et discours, Collection Praxilingue, Université Montpellier 3, 69-89.

BOUCHARD, R. (2004 a) : Cours magistral et « sources de savoir » : médiation discursive et médiation grammaticale, in Delamotte-Legrand, R. (dir.) Les médiations langagières, Des discours aux acteurs sociaux, vol. II, Dyalang, CNRS, Publications de l'université de Rouen, 243-254.

BOUCHARD, R. (2004 b) : Les inter-actions didactiques, un défi pour l'analyse du discours ? Étude du rôle des « artefacts » dans une inter-action de correction collective (enseignement de géométrie au collège), in Auchlin, A. (et al.) (dir.) Structures et discours, Québec, Éd Nota Bene, 45-66.

BOUCHARD, R. (2004 c) : Narration, actions et objets : étude de transactions didactiques dilogales en

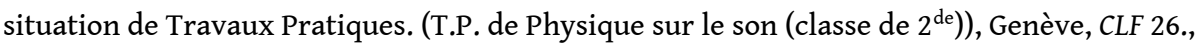
293-320.

Lidil, 31 | 2005 
BOUCHARD, R., ROLET, C. (2003) : Pour une méthodologie d'analyse didactico-interactionnelle des pratiques d'enseignement/apprentissage : A propos d'une séance de mathématiques à l'école primaire, in Jaubert, M., Rebière, M. \& Bernié, J.-P., Construction des connaissances et langage dans les disciplines d'enseignement, CD-Rom, Iufm d'Aquitaine et Université Bordeaux 2.

BOUCHARD, R., PARPETTE, C., POCHARD, J.-C. (2005) : Le cours magistral et son double, le polycopié : relations et problématique de réception en L2, in Mochet, M.-A. (ed.) Pluralité des langues et pluralité des supports, Lyon, Presses de l'ENS Lsh.

CAPLOW, T. (1971) : Deux contre un, ESF.

DABÈNE, L. (1984) : Communication et métacommunication en classe de langue, in Bouchard, R. (dir.) Les échanges langagiers en classe de langue, Grenoble, Ellug, 129-138

FILLIETTAZ, L. (2002) : La parole en action. Éléments de pragmatique psycho-sociale, Québec, Éd. Nota Bene.

KERBRAT-ORECCHIONI, C. (1990 a) : L'énonciation : de la subjectivité dans le langage, Paris, A. Colin.

KERBRAT-ORECCHIONI, C. (1990 b) : Les interactions verbales, Paris, A. Colin.

KERBRAT-ORECCHIONI, C. (2004) : Introducing polylogue, Journal of Pragmatics, 36, 1-24.

KeRBRAT-ORECCHIONI, C., PLANTIN, C. (eds.) (1995) : Le trilogue, Lyon, PUL.

NOYAU, C., QUASHIE, M. (2004) : L'école et la classe comme environnement écologique, in Defays, J.M. (eds.) Les didactiques du français un prisme irisé, EME, Cortil-Wodon.

MEHAN, H. (1978) : Learning lessons: social organization of the classroom. Cambridge (MA), Harvard University Press.

RABATEL, A. (2004) : Déséquilibres interactionnels et cognitifs, postures énonciatives et coconstruction des savoirs : co-énonciateurs, sur-énonciateurs et archi-énonciateurs, in Rabatel, A. (ed.) Interactions orales en contexte didactique, Lyon, PUL., 29-66.

ROULET, E. et al (1985) : L'articulation du discours en français contemporain, Berne, P. Lang. SCHUBAUER-LEONI, M.-L. (1997) : Interactions didactiques et interactions sociales : quels phénomènes et quelles constructions conceptuelles, Skholé. Cahiers de la recherche et $d u$ développement $\mathrm{n}^{\circ}$ 7, Iufm Aix-Marseille, 103-134.

SENSEVY, G., QUILLIO, S. (2002) : Les discours du professeur. Vers une pragmatique didactique, Revue française de pédagogie, 146, 47-56.

SINCLAIR, J.M., COULTHARD, R.M. (1975) : Towards an Analysis of Discourse, Oxford, OUP.

VERNANT, D. (1997) : Du discours à l'action, Paris, PUF.

WitKo-commeAu, A. (1995) : Du trilogue dans le polylogue, in Kerbrat-Orecchioni, C. et Plantin, C. (eds.) Le trilogue, Lyon, PUL, 284-306.

\section{NOTES}

1. Après les théories de l'énonciation, la pragmatique des actes de parole et la pragmatique conversationnelle. 
2. Nous parlons même volontiers à propos des interactions de classe de polylogues praxéologiques, inégaux, ritualisés, en ajoutant ainsi deux facettes supplémentaires qui ne pourrront qu'être évoquées encore plus superficiellement dans cet article.

3. Corpus d'histoire et de mathématiques en 6e recueillis par M. Frangin (Mémoire de DEA de Sciences du langage, Lyon 2, 2002).

4. Il est alors nécessaire de distinguer non seulement les différents locuteurs/acteurs mais aussi l'(es) interlocuteur(s) au(x)quel(s) ils s'adressent (Bouchard \& Rolet, 2003).

\section{AUTEUR}

ROBERT BOUCHARD

Université Lumière-Lyon 2, Laboratoire ICAR (UMR 5191) 